This is the accepted version of the following article: Kikelj M, Lipovšek B, Bokalič M, Topič M. Spatially resolved electrical modelling of cracks and other inhomogeneities in crystalline silicon solar cells. Progress in Photovoltaics: Research and Applications 2021; 29, pp. 124-133. DOI:10.1002/pip.3348, which has been published in final form at https://doi.org/10.1002/pip.3348. This article may be used for non-commercial purposes in accordance with the Wiley SelfArchiving Policy [http://www.wileyauthors.com/self-archiving]. 


\title{
Spatially Resolved Electrical Modelling of Cracks and Other Inhomogeneities in Crystalline Silicon Solar Cells
}

\author{
Miha Kikelj ${ }^{1 *}$, Benjamin Lipovšek ${ }^{1}$, Matevž Bokalič ${ }^{1}$, Marko Topič ${ }^{1}$ \\ ${ }^{1}$ University of Ljubljana, Faculty of Electrical Engineering, Tržaška cesta 25, SI-1000 Ljubljana, \\ Slovenia
}

\begin{abstract}
We developed a fully scalable quasi-SPICE approach for electrical modelling of stress induced inhomogeneities in crystalline silicon solar cells, based on damage detected by electroluminescence imaging. We present a model of active and inactive micro-cracks, highlight their effects on the current-voltage characteristic, and validate the model on a number of experimental cases. The generalisation of our model to a complexly damaged solar cell shows excellent agreement with the measurements, with only $0.12 \%$ estimation error of the power loss of the complexly damaged cell. The ability to accurately model fundamental stress induced inhomogeneities and their effects on cell level is crucial to estimating long-term performance degradation and energy yield of installed photovoltaic modules throughout their lifetime. The developed scalable model paves a path to intricate, statistically supported modelling for power loss prediction and mitigation in solar cells, photovoltaic modules and photovoltaic arrays caused by mechanically induced inhomogeneities.
\end{abstract}

\section{KEYWORDS}

micro-cracks, stress induced inhomogeneities, modelling, EL, power loss evaluation

\section{* Correspondence}

Miha Kikelj, University of Ljubljana, Faculty of Electrical Engineering, Tržaška cesta 25, SI-1000 Ljubljana, Slovenia

E-mail: miha.kikelj@fe.uni-lj.si 


\section{INTRODUCTION}

The market for photovoltaics (PV) has experienced a steady growth in recent years, with cumulative installed PV capacity reaching $400 \mathrm{GWp}$ in 2017 [1], $500 \mathrm{GWp}$ in 2018 [2] and it is expected to reach 1 TWp installed capacity by 2022/23 [2], with crystalline silicon (c-Si) solar cells and PV modules taking the majority of the share [1]. With photovoltaic energy systems reaching terawatt-scales, even the slightest decrease in solar cell performance could potentially lead to a loss of thousands of GWh of global annual energy yield. Studies from previous work have shown that micro-cracks, which do not affect contact fingers or busbars, could lead to a power loss of up to $2.5 \%$ on module level [3], while more extensively damaged modules could exhibit a power loss of up to $20 \%$ [4], [5]. Taking that into concern, by 2022, we could be losing up to nearly $300 \mathrm{TWh}$ of annual energy yield solely due to damaged modules, which is just shy of $1 \%$ of Europe's annual electric energy consumption and slightly less than 50\% of Germany's in 2017 [6]. Therefore, the ability to model fundamental stress induced inhomogeneities caused by different environmental factors (wind, snow, thermal expansion), and their effects on cell level is crucial to estimating longterm performance degradation and making design changes to possibly mitigate these power losses. At the same time, the scale of impact urges for such papers that elucidate the problem and serve as awareness raising of the importance in handling of PV modules during transport and installation as well as mitigation measures for in-field operation conditions of mechanical stresses.

From the modelling perspective, much has been done in the field of understanding the formation of cracks and their effects on cell and module level. An extensive study performed by M. Paggi et al. [7], takes into account the crystalline structure of a PV solar cell and performs a stress analysis of a small module predicting the formation of cracks and possible inactive cell areas. From the obtained results it employs a one-diode model for each cell, reducing the photo-generated current by the ratio between the active and the inactive cell areas and thus achieving a drop in the generated power. Other approaches are presented by M. Köntges et al. [3] and T. Kropp et al. [4], who argue that cracking does not influence the p-n junction but merely disconnects certain areas and introduces additional series resistances to the damaged areas. The only main difference is that the former approach separates the cell only by active to inactive cell area ratio, while the latter allows

for multiple separate inactive cell areas each connected by their own series resistances. With this 
approach, T. Kropp et al. [4] claim to have been able to reach performance estimation with an accuracy better than $1 \%$ in the maximum power point.

However, all aforementioned approaches partially neglect the geometry of the mechanical faults and solar cells themselves, employing integral parameters to describe the effects of inhomogeneities. Our approach, based on defects detected by means of electroluminescence (EL) imaging, on the other hand, utilises an open-source photovoltaic simulator PVMOS [8] to accurately, and in a similarly structured manner as presented by R. Kimovec et al. [9], model mechanical defects and their influences on the current-voltage $(I-V)$ characteristics including cracks' geometry and their relative position within the cell. Instead of integral parameters we only change local parameters in the part of the cell where the damage has occurred, without introducing any additional ones. This approach allows us to evaluate the effects of inhomogeneities in a bottomup, scalable fashion, from parts of a cell to cells, modules and module arrays or even whole PV power plants.

\section{MODELLING}

\subsection{Modelling a cell - PVMOS}

We employed a freely available 2.5D quasi-SPICE finite-difference photovoltaic simulator PVMOS [8] to model our damaged cells. The model consists of a layer stack of planar networks as shown in Fig. 1.a, each layer modelling a separate layer in a solar cell. In our case, for a soldered sample cell, we used 4 layers to describe the top ribbon connections $\left(L_{1}\right)$, the top metallisation (busbars and fingers $-L_{2}$ ), and the c-Si part of the cell including the bottom metallisation and the bottom ribbon ( $L_{3}$ and $L_{4}$ ). $L_{3}$ models the emitter layer of the cell connected via a p-n junction to $L_{4}$ modelling the structured sheet resistance of the bulk and the bottom metallisation. Each of the layers is characterised by its sheet resistance and is vertically connected to adjacent layers via an either resistive contact (modelling contact resistance), a p-n junction modelled by a one- or twodiode model or a junction implementing an arbitrary $J-V$ characteristic. Furthermore, a cell is horizontally subdivided into areas, each with its own set of properties for layer sheet resistances and their interconnections, allowing us to pattern the cell to implement busbars and fingers, and most importantly it allows us to input exact geometry of stress induced inhomogeneities. Described 
areas are further discretised in a finite-difference fashion producing smaller rectangular areas which can be visualised as shown in Fig. 1.b.

The resistance $R_{r b}$ in the layer $L_{l}$ represents the sheet resistance of the ribbon, and $R_{i s o}$ an "infinite" isolation sheet resistance, modelling a disconnection from the adjacent nodes. Both $R_{b}$ and $R_{i s o}$ are vertically connected to the layer $L_{2}$ through the contact resistance $R_{c o n}$. The resistance $R_{f}$ in $L_{2}$ models the sheet resistance of the screen-printed front fingers and busbars, which are again vertically connected to the layer $L_{3}$ via $R_{c o n}$. The resistance $R_{e}$ in $L_{3}$ represents the sheet resistance of the emitter layer. The connection between $L_{3}$ and $L_{4}$ in Fig. 1.a is represented by a two-diode model (TWDM) shown in Fig. 1.c, thus modelling a p-n junction between the emitter and the bulk layers. The bulk layer is described by resistance $R_{b}$, which is a structured sheet resistance of the bulk and the bottom aluminium metallisation $R_{\text {alu }}$. The parameters of the two-diode model were obtained by calibrating the TWDM to a dark $I-V$ characteristic of a healthy, undamaged cell, where series resistance was left out and set to a minimum value, since its effects are described by distributed resistances of the other layers. We also calibrated the sheet resistance of the front metallisation $R_{f}$ and the sheet resistance of the emitter layer $R_{e}$ to match the trends of cross-finger and cross-busbar profiles in the measured EL image. Sheet resistances modelling the top and bottom ribbon $R_{r b}$ and the bottom metallisation $R_{a l u}$ were determined from literature, while $R_{c o n}$ and $R_{\text {iso }}$ were, for simplicity, set to their minimal and maximal values respectively allowed by the simulator. A short summary of the parameters is given in Table 1, and specific values of all parameters in Table 2 in section 3.1.

The set-up model is solved for node voltages and currents in all three directions as well as for the integral $I-V$ characteristic allowing for cell and sub-cell result evaluation. In the following sections the modelling approach described above will be used to model the effects of solar cells' inhomogeneities.

\subsection{Classification of stress-induced inhomogeneities}

We have studied four types of stress induced inhomogeneities of multicrystalline solar cells and classified them into categories according to their influence on the EL image. Examples and their classification according to IEC 60904-13 [10] are shown in Fig. 2, while detailed explanation follows in the next sections. Inactive cracks (Fig. 2.a) are visible darker lines in the EL image, 
usually not observable by mere naked eye inspection of a solar cell. They do not result in a global change in the EL intensity profile. Active cracks (Fig. 2.b) exhibit a sharp, step-like change in EL intensity. Larger dark areas, roughly the same intensity as the busbars, are classified as dead cell areas (Fig. 2.c), and darker areas around the fingers, ranging from slight decrease in EL intensity to completely dark areas, are a signal of contact finger interruption - CFI (Fig. 2.d).

Despite the fact that our method is able to simulate any of the previously mentioned faults, we have, in the scope of this work, experimentally addressed, validated and quantitatively assessed the phenomenon of inactive cracks, although all types of defects are included in the application of the model to a complexly damaged solar cell.

\subsection{Modelling inactive cracks}

As visible from Fig. 2.a, inactive cracks have no influence on the overall EL profile but affect the EL intensity only in the vicinity of the crack. In such case the EL profile along the fingers follows the trend of the exponential of a normally quadratic junction voltage profile [11], while it exhibits a drop when crossing the crack as shown in Fig. 3.a. According to the observed EL intensities from samples diagnosed with inactive cracks, EL physical background and research work done by J. Käsewieter et al. [12], who examined resistances of the front and the back metallisation during mechanical loading of a solar cell, and SEM images of a crack in a solar cell by T. M. Pletzer et al. [13], we established that inactive cracks represent only damage (discontinuity) in the silicon crystal structure (i.e. the p-n junction and the bulk), while no major changes occur in neither front nor back metallisation. The schematic of this type of cracks is shown in Fig. 3.c.

With the aforementioned extent of crack damage and measured influences of the inactive cracks on the dark $I-V$ curve, we introduced local changes in the cracked cell's PVMOS model to recreate the actual measured $I-V$ characteristic. Since cracking disturbs the crystal lattice, it introduces additional recombination in the area of the crack, accounting for the mid-voltage increase in the dark $I-V$ curve. We therefore locally (solely in the area of the crack) increased the reverse saturation current density $J_{02}$ of the second diode in the TWDM connecting $L_{3}$ and $L_{4}$. Additionally, locally decreasing the shunt resistance of the TWDM (between $L_{3}$ and $L_{4}$ ) accounts for the increase of the absolute value of the reverse current in the dark $I-V$ characteristic, which is not sufficiently covered by an increase in $J_{02}$. The effects of inactive cracks on the dark $I-V$ curve are shown in Fig 3.d. 


\subsection{Modelling active cracks}

Active cracks, on the other hand, do introduce a significant change in the EL profile. A normally monotonous profile exhibits the same kind of a drop as in the case of inactive cracks, except that the profile does not recover to the previous value after the crack but continues from a lower value. An example of an active crack's EL profile is shown in Fig. 3.b. An explanation of the stepwise decrease in EL intensity should lie in an increased series resistance of either front or back metallisation. Since disconnection of the front metal fingers usually results in a well-defined darkened area around the fingers [14] (as visible in Fig. 2.d), we expect that an active crack, besides damaging the silicon crystal structure, damages the bottom metallisation layer as well, and thus locally increases its sheet resistance. According to [12], the back metallisation exposed to repetitive stress deforms plastically, thus leading to a permanent change in its sheet resistance. The extent of the expected active crack damage is shown in Fig 3.c. On this basis we have upgraded the PVMOS crack model to include, besides changes to the TWDM (described in the previous section), local increases in the back-metallisation's sheet resistance modelled by $R_{b}$ in the bottom layer $\left(L_{4}\right)$, thus partially disconnecting the affected part of the cell.

\subsection{Experimental model calibration}

Firstly, we had to establish a baseline for further measurements and simulations. Dark $I-V$ curve measurements and EL measurements were performed on a bare, undamaged 1/8 sample cell, similar to the $156 \times 156 \mathrm{~mm}^{2}$ multicrystalline solar cells used in a 2010 Bisol BMU 233 PV module. We also performed a geometric feature extraction layer by layer to extract the size of the cell, shape of the front and the back metallisation, and other cell-specific features and recreated them as feature masks for further use with PVMOS. To determine key unknown cell parameters like emitter sheet resistance $R_{e}$, contact finger sheet resistance $R_{f}$ and the parameters of the TWDM modelling the p$\mathrm{n}$ junction, we performed a two stage iterative EL and $I-V$ matching process, similar to the process in [15]. We matched the measured and the simulated dark $I-V$ curves, while also paying attention to the match between the measured and the simulated EL profiles along and perpendicular to the busbars at the front of the cell. Using the approach above, we derived a PVMOS model describing an undamaged cell. Fig. 4 shows the experimental workflow for the calibration procedure. 
In the next stage we progressively cracked the non-encapsulated sample cell and modelled each iteration with a corresponding PVMOS model. The cracking procedure consisted of flipping the cell backside-up, with one of the edges slightly lifted, and then pressing down on the back metallisation until a crack occurred. The elasticity of the front metallisation allowed for the damage to be contained only to the silicon layer of the cell. Bending it the other way, firstly damaged the bottom metallisation and the shockwave then propagated through the cell, damaging the $p-n$

junction and severing the fingers. We then verified the crack type by means of EL and measured the sample cell's dark $I-V$ curve. The same cell was then cracked repeatedly in order to study the cumulative effect of cracks on the $I-V$ curve.

The geometry of a fresh crack at each stage was recreated, appended to the previously simulated model and the parameters within the cracked area were optimised until a satisfactory match between the measured and the simulated dark $I-V$ curves and EL profiles was achieved. Obtained crack parameters were then used as a starting point for the crack model in the next cracking stage. At this point only samples where the previous crack has not changed or propagated were considered for further evaluation. Again, the parameters of the newly modelled crack were changed until a match was achieved while the parameters of all previous cracks were left unchanged. Utilising this approach, we were able to model small cracked sample cells along with an already complexly damaged full-sized cell, described in detail in the following sections.

\section{RESULTS AND DISCUSSION}

We verified the model on a crack-by-crack, sample cell level for the case of inactive cracks, described in section 3.1. We then applied the model, which also includes other stress induced inhomogeneities to a complexly damaged full-sized $\left(156 \times 156 \mathrm{~mm}^{2}\right)$ cell to study the cumulative effect of a variety of stress induced inhomogeneities, described in section 3.2.

\subsection{Inactive crack verification}

\subsubsection{Healthy cell}

In the first step we calibrated the model of a healthy cell against a healthy sample cell's dark $I-V$ curve, as explained in section 2.5. The measured and simulated $I-V$ curves shown in Fig. 5.a agree fairly well, displaying a nearly perfect match for the voltages around $0.6 \mathrm{~V}$ and in the reverse bias 
region. One can also observe a similarly good agreement of the measured and the simulated average EL profiles in both directions (Fig. 5.c and 5.d).

There is, however, a slight discrepancy between the two $I-V$ curves in the lower two thirds of the forward bias, as well as a slight mismatch between the measured and the simulated EL profiles, which we have attributed to the simplifications of the model.

The cell was modelled as a homogeneous entity without areas of enhanced recombination such as grain boundaries and dislocations, visible as darker parts in the measured EL image in Fig. 5.b, which should increase the current in the lower part of the forward bias. The same reasoning applies to the fluctuations of the measured EL profiles (Fig. 5.c and 5.d). Because the areas of enhanced recombination are not modelled, the modelled cell exhibits, on average, a higher reverse saturation current density $J_{02}$, which leads to higher current densities at lower voltages. Since EL imaging was performed at a constant short circuit current $I_{s c}$ injection level, higher current density of $J_{02}$ would manifest as a lower overall voltage and thus lower simulated EL intensities, explaining the offset between the profiles in Fig. 5.c. Despite the offset of the simulated EL profile, we are convinced that the model provides a suitable description of the device, since the trends of EL profiles (Fig. 5.c and 5.d) closely follow the measurements. This calibrated model was later used in the evaluations of the effects of inactive cracks. The model's parameters used in PVMOS simulations are shown in Table 2.

Another interesting difference between the EL profiles is visible around the busbar (in the middle of Fig. 5.c), where, in the simulated case the EL intensity spikes up and is then shaded by the busbar itself, the measurement exhibits a different trend. Instead of an expected rise, the EL intensity actually decreases. Additional experiments with an unsoldered sample cell produced the same trends and have thus eliminated the possibility that the change in the profile could be linked to soldering induced microcracking. As the width of the area exactly corresponds to the width of the busbar at the back of the cell, we expect that the difference could be due to different optical properties of the busbar region, but the hypothesis is still a subject of further research. We attributed the differences at the beginning and the end of the cross-finger EL profile (Fig. 5.d) to edge recombination, which was also not modelled in this scope. 


\subsubsection{Damaged cell}

The same sample cell was then damaged progressively, as explained in section 2.5, and its EL images and dark $I-V$ curves were measured in every iteration, with Fig. 6.a showing EL images of the cracking progression while the changes in the dark $I-V$ curves are shown in Fig. 6.b. As one can observe from Fig. 6.b, progressive cracking leads to an increase in the reverse bias current and an increase in the current in the $0.4 \mathrm{~V}$ range in the forward bias region as assumed in section 2.3.

We have extracted the cracks' geometry and used it to simulate separate cracking stages. According to the assumptions on crack influences established in sections 2.3 and 2.4 we changed the local values of $J_{02}$ and $R_{s h}$ of the TWDM, thus modelling forward and reverse bias current changes respectively. Fig. 7.a and 7.b show the simulated EL images and the dark $I-V$ curves of the progressively cracked cell, while model values used in the simulations are shown in Table 3.

The measured cross-busbar EL profiles are presented in Fig. 8.a. As expected, EL intensity decreases in the vicinity of the cracks, while the overall drop in the mean value of the profiles throughout the cracking procedure could be accounted for by the drop of junction voltage due to increasing cell temperature throughout the experiment. In the case of simulated profiles shown in Fig. 8.b, one can clearly observe the same EL profile trends around the cracked areas, where the profile takes a sudden dip but afterwards recovers to the previous value following the EL intensity decrease away from the busbar.

The results presented in Fig. 6.b and 7.b demonstrate that the influence of the progressive inactive crack formation, namely the trend of current increase observed in the measured dark $I-V$ curves, were reproduced accurately by simulations. A similarly good match of the trends is also observed in the measured and the simulated EL profiles presented in Fig. 8, while the small discrepancies in the absolute values can mainly be attributed to the aforementioned simplifications of the model that treat the other regions of the cell as perfectly homogeneous.

\subsection{Application example}

The approach that we described in the previous sections can be used to model complexly damaged solar cells including multiple inhomogeneities of various types. In this contribution we have measured and modelled a cell from a 2010 Bisol BMU 233 PV module from our test site in 
Ljubljana. We have then compared the results to those of a healthy cell of the same module to observe the effects of stress induced damage.

We measured a dark and light (at STC conditions) $I-V$ curve of a healthy cell and then again measured the damaged cell in the same manner. From the EL image of the damaged cell shown in Fig. 9.a we extracted the stress induced inhomogeneities' geometry and type, as shown in Fig. 9.b. Extracted geometry was then used to simulate the EL intensity of the damaged cell, shown in Fig. 9.c, as well as the light $I-V$ curves which are shown in Fig. 9.d for both measurement and simulation cases. From the $I-V$ curves, we finally estimated the power loss that can be attributed to the stress induced inhomogeneities.

The comparison between the measured and the simulated EL images (Fig. 9.a and Fig. 9.b) and light $I-V$ curves presented in Fig. 9.d again exhibit a very good agreement, despite the fact that, in this case, we simulated a much more extensively damaged solar cell. We successfully modelled a combined influence of active and inactive cracks as well as contact finger interruptions - CFI, which further confirms the validity and applicability of the developed modelling approach.

Finally, in terms of power generation, the measured damaged cell exhibits a 5.7\% power drop in the maximum power point (MPP) compared to a healthy cell, which corresponds well with the values established in [3], [4] and [5]. In the case of the simulated sample cell we estimated the power drop in the MPP of the damaged cell with an error of mere $0.12 \%$ compared to the measurements.

With the ability to accurately simulate the operating conditions of extensively damaged solar cells one could easily extend the study for accurate power prediction of damaged PV modules, PV module arrays and systems using the same bottom-up approach. Further on, the model could be expanded in a similar fashion to include other failures and inhomogeneities, areas of enhanced recombination (material impurities), or even differences in cell temperature and local hotspots. For example, one could include the effects of solder joint or solder to silver paste cracks [16], [17] as properly increased contact resistance between the top ribbon connections $L_{1}$ and the top metallisation layer $L_{2}$ (busbars and fingers). While these effects were not the subject of present research and would have been an unnecessary complication for this contribution, their inclusion would yield an even more accurate description of the cell. The aforementioned effects, which can 
significantly influence the PV devices' performance, could and are to be taken into account in future work.

\section{CONCLUSIONS}

This work ventures into the understanding of cracks' and other inhomogeneities' manifestation in solar cells as a consequence of exposure to mechanical stress. We developed a modelling approach based on the PVMOS 2.5D workflow that accurately describes each individual inhomogeneity and evaluates its impact on performance of a solar cell in a bottom-up, scalable fashion. We studied the influence of individual inactive cracks, active cracks, finger disconnections and dead cell areas on the EL profile. We calibrated and verified the approach on a number of experimental cases, showing good agreement between the measured and simulated $I$ - $V$ curves and EL intensity profiles. The approach could be easily extended to model other inhomogeneities (e.g. grain boundaries, edge recombination). We also demonstrated the scalability and applicability of our approach on a fullsized complexly damaged cell, that exhibited a combination of the aforementioned inhomogeneities. With our approach, we were able to estimate the power loss of the complexly damaged cell with $0.12 \%$ error compared to the measurements.

With the demonstrated ability to accurately simulate the performance of arbitrarily damaged solar cells we could employ a structural stress analysis as performed by M. Paggi et al. [7] combined with module stress analysis, principal direction and fracture probability as evaluated by F. Haase et. al [18], M. Köntges et al. [19] or M. Sander et al. [20], and a statistical study of location dependent damage manifestation in order to build an intricate model for degradation prediction due to mechanically induced inhomogeneities. With such a model further actions could be taken in solar cell, module or perhaps even power plant design to minimise degradation and energy yield loss over the modules' lifetime and thus give a long-term boost to an already growing PV market.

\section{ACKNOWLEDGMENTS}

Authors gratefully acknowledge funding from the Slovenian Research Agency under the research programme P2-0197 and the European Union's Horizon 2020 programme in the framework of the project "HighLite" under GA No. 857793. The authors thank Kristijan Brecl and Rok Kimovec for useful discussions. 


\section{REFERENCES}

[1] F. ISE, 'Photovoltaics Report'.

[2] N. M. Haegel et al., 'Terawatt-scale photovoltaics: Transform global energy', Science, vol. 364, no. 6443, pp. 836-838, May 2019, doi: 10.1126/science.aaw1845.

[3] M. Köntges, I. Kunze, S. Kajari-Schröder, X. Breitenmoser, and B. Bjørneklett, 'The risk of power loss in crystalline silicon based photovoltaic modules due to micro-cracks', Solar Energy Materials and Solar Cells, vol. 95, no. 4, pp. 1131-1137, Apr. 2011, doi: 10.1016/j.solmat.2010.10.034.

[4] T. Kropp, M. Schubert, and J. H. Werner, 'Quantitative Prediction of Power Loss for Damaged Photovoltaic Modules Using Electroluminescence’, Energies, vol. 11, no. 5, Art. no. 5, May 2018, doi: 10.3390/en11051172.

[5] M. Dhimish, V. Holmes, M. Dales, and B. Mehrdadi, 'Effect of micro cracks on photovoltaic output power: case study based on real time long term data measurements', Micro Nano Letters, vol. 12, no. 10, pp. 803-807, 2017, doi: $10.1049 / \mathrm{mnl} .2017 .0205$.

[6] IEA, 'Key World Energy Statistics 2019'.

[7] M. Paggi, M. Corrado, and M. A. Rodriguez, 'A multi-physics and multi-scale numerical approach to microcracking and power-loss in photovoltaic modules', Composite Structures, vol. 95, pp. 630-638, Jan. 2013, doi: 10.1016/j.compstruct.2012.08.014.

[8] B. E. Pieters, 'A free and open source finite-difference simulation tool for solar modules', in 2014 IEEE 40th Photovoltaic Specialist Conference (PVSC), Jun. 2014, pp. 1370-1375, doi: 10.1109/PVSC.2014.6925173.

[9] R. Kimovec, H. Helmers, A. W. Bett, and M. Topič, 'Comprehensive electrical loss analysis of monolithic interconnected multi-segment laser power converters', Progress in Photovoltaics: Research and Applications, vol. 27, no. 3, pp. 199-209, 2019, doi: 10.1002/pip.3075.

[10] 'IEC TS 60904-13'. 2017.

[11] K. Bothe et al., 'Electroluminescence Imaging as an In-Line Characterisation Tool For Solar Cell Production', Dresden, 2006, p. 5.

[12] J. Käsewieter, F. Haase, M. H. Larrodé, and M. Köntges, 'Cracks in Solar Cell Metallization Leading to Module Power Loss under Mechanical Loads', Energy Procedia, vol. 55, pp. 469-477, Jan. 2014, doi: 10.1016/j.egypro.2014.08.011.

[13] T. M. Pletzer, J. I. van Mölken, S. Rißland, O. Breitenstein, and J. Knoch, 'Influence of cracks on the local current-voltage parameters of silicon solar cells', Progress in Photovoltaics: Research and Applications, vol. 23, no. 4, pp. 428-436, 2015, doi: 10.1002/pip.2443.

[14] I. Zafirovska, M. K. Juhl, J. W. Weber, J. Wong, and T. Trupke, 'Detection of Finger Interruptions in Silicon Solar Cells Using Line Scan Photoluminescence Imaging', IEEE Journal of Photovoltaics, vol. 7, no. 6, pp. 1496-1502, Nov. 2017, doi: 10.1109/JPHOTOV.2017.2732220.

[15] M. Bokalic and M. Topic, Spatially Resolved Characterization in Thin-Film Photovoltaics. Springer International Publishing, 2015.

[16] U. Itoh, M. Yoshida, H. Tokuhisa, K. Takeuchi, and Y. Takemura, 'Solder Joint Failure Modes in the Conventional Crystalline Si Module', Energy Procedia, vol. 55, pp. 464-468, Jan. 2014, doi: 10.1016/j.egypro.2014.08.010.

[17] J.-S. Jeong, N. Park, and C. Han, 'Field failure mechanism study of solder interconnection for crystalline silicon photovoltaic module', Microelectronics Reliability, vol. 52, no. 9, pp. 2326-2330, Sep. 2012, doi: 10.1016/j.microrel.2012.06.027.

[18] F. Haase, J. Käsewieter, S. R. Nabavi, E. Jansen, R. Rolfes, and M. Köntges, 'Fracture Probability, Crack Patterns, and Crack Widths of Multicrystalline Silicon Solar Cells in PV Modules During Mechanical Loading', IEEE Journal of Photovoltaics, vol. 8, no. 6, pp. 1510-1524, Nov. 2018, doi: 10.1109/JPHOTOV.2018.2871338.

[19] U. Jahn, I. Kunze, S. Kajari-Schröder, and M. Köntges, 'Crack Statistic of Crystalline Silicon Photovoltaic Modules', 26th European Photovoltaic Solar Energy Conference and Exhibition, pp. 3290-3294, Oct. 2011, doi: 10.4229/26thEUPVSEC2011-4EO.3.6. 
[20] M. Sander, S. Dietrich, M. Pander, M. Ebert, and J. Bagdahn, 'Systematic investigation of cracks in encapsulated solar cells after mechanical loading', Solar Energy Materials and Solar Cells, vol. 111, pp. 8289, Apr. 2013, doi: 10.1016/j.solmat.2012.12.031. 


\section{TABLES}

\begin{tabular}{clc}
\hline \hline Symbol & Description & Unit \\
\hline $\boldsymbol{R}_{\boldsymbol{r b}}$ & Sheet resistance of the copper ribbon & {$[\Omega / \square]$} \\
$\boldsymbol{R}_{\boldsymbol{f}}$ & Sheet resistance of the screen-printed fingers and busbars & {$[\Omega / \square]$} \\
$\boldsymbol{R}_{\boldsymbol{e}}$ & Sheet resistance of the emitter & {$[\Omega / \square]$} \\
$\boldsymbol{R}_{\boldsymbol{b}}$ & Sheet resistance of the bulk & {$[\Omega / \square]$} \\
$\boldsymbol{R}_{\boldsymbol{a l u}}$ & Sheet resistance of the aluminium back metallisation & {$[\Omega / \square]$} \\
$\boldsymbol{R}_{\boldsymbol{i s o}}$ & Isolation sheet resistance modelling a disconnection & {$[\Omega / \square]$} \\
$\boldsymbol{R}_{\boldsymbol{c o n}}$ & Contact resistance between layers & {$\left[\Omega \mathrm{cm}^{2}\right]$} \\
\hline \hline & \multicolumn{1}{c}{ Two-diode Model Parameters } \\
$\boldsymbol{J}_{\boldsymbol{0} \boldsymbol{1}}$ & Reverse saturation current density of the diode $D_{1}$ & {$\left[\mathrm{~A} / \mathrm{cm}^{2}\right]$} \\
$\boldsymbol{n}_{\boldsymbol{1}}$ & Ideality factor of the diode $D_{1}$ & $/$ \\
$\boldsymbol{J}_{\boldsymbol{0} \mathbf{2}}$ & Reverse saturation current density of the diode $D_{2}$ & {$\left[\mathrm{~A} / \mathrm{cm}^{2}\right]$} \\
$\boldsymbol{n}_{\mathbf{2}}$ & Ideality factor of the diode $D_{2}$ & $/$ \\
$\boldsymbol{R}_{\boldsymbol{s} \boldsymbol{h}}$ & Shunt resistance of the two-diode model & {$\left[\Omega \mathrm{cm}^{2}\right]$} \\
$\boldsymbol{R}_{\boldsymbol{s}}$ & Series resistance of the two-diode model & {$\left[\Omega \mathrm{cm}^{2}\right]$} \\
\hline \hline
\end{tabular}

Table 1. Description of symbols and quantities used.

\begin{tabular}{llllll}
\hline \hline \multicolumn{7}{l}{ Two-diode Model Parameters } \\
\hline $\boldsymbol{J}_{\boldsymbol{0} 1}\left[\mathrm{~A} / \mathrm{cm}^{2}\right]$ & $\boldsymbol{n}_{\boldsymbol{1}}$ & $\boldsymbol{J}_{\boldsymbol{0} 2}\left[\mathrm{~A} / \mathrm{cm}^{2}\right]$ & $\boldsymbol{n}_{2}$ & $\boldsymbol{R}_{\boldsymbol{s h}}\left[\Omega \mathrm{cm}^{2}\right]$ & $\boldsymbol{R}_{s}\left[\Omega \mathrm{cm}^{2}\right]$ \\
$1.24 \mathrm{e}-12$ & 1 & $5.88 \mathrm{e}-8$ & 2 & $5.79 \mathrm{e} 5$ & $1 \mathrm{e}-10$ \\
\hline \hline Other Cell Parameters & & & \\
\hline $\boldsymbol{R}_{\boldsymbol{f}}$ & $\boldsymbol{R}_{e}[\Omega / \square]$ & $\boldsymbol{R}_{b}[\Omega / \square]$ & $\boldsymbol{R}_{\text {alu }}[\Omega / \square]$ & $\boldsymbol{R}_{\text {con }}\left[\Omega \mathrm{cm}^{2}\right]$ & $\boldsymbol{R}_{\text {iso }}[\Omega / \square]$ \\
$1.6 \mathrm{e}-3$ & 35 & 30 & $1.16 \mathrm{e}-2$ & $1 \mathrm{e}-10$ & $1 \mathrm{e} 10$ \\
\hline \hline
\end{tabular}

Table 2. PVMOS parameters used in the simulations of the sample cells.

\begin{tabular}{lcccccc}
\hline & $\begin{array}{c}\boldsymbol{J}_{\boldsymbol{0 1}} \\
{\left[\mathrm{A} / \mathrm{cm}^{2}\right]}\end{array}$ & $\boldsymbol{n}_{\boldsymbol{1}}$ & $\begin{array}{c}\boldsymbol{J}_{\boldsymbol{0} 2} \\
{\left[\mathrm{~A} / \mathrm{cm}^{2}\right]}\end{array}$ & $\boldsymbol{n}_{\boldsymbol{2}}$ & $\begin{array}{c}\boldsymbol{R}_{\boldsymbol{s}} \\
{\left[\Omega \mathrm{cm}^{2}\right]}\end{array}$ & $\begin{array}{c}\boldsymbol{R}_{\boldsymbol{s} \boldsymbol{h}} \\
{\left[\Omega \mathrm{cm}^{2}\right]}\end{array}$ \\
\hline Crack 1 & $1.24 \mathrm{e}-12$ & 1 & $4.55 \mathrm{e}-6$ & 2 & $1 \mathrm{e}-10$ & $5.3 \mathrm{e} 3$ \\
Crack 2 & $1.24 \mathrm{e}-12$ & 1 & $8.55 \mathrm{e}-6$ & 2 & $1 \mathrm{e}-10$ & $4.2 \mathrm{e} 3$ \\
\hline \hline
\end{tabular}

Table 3. Cracks' local parameters used in the simulations of the damaged cells. 


\section{FIGURES}

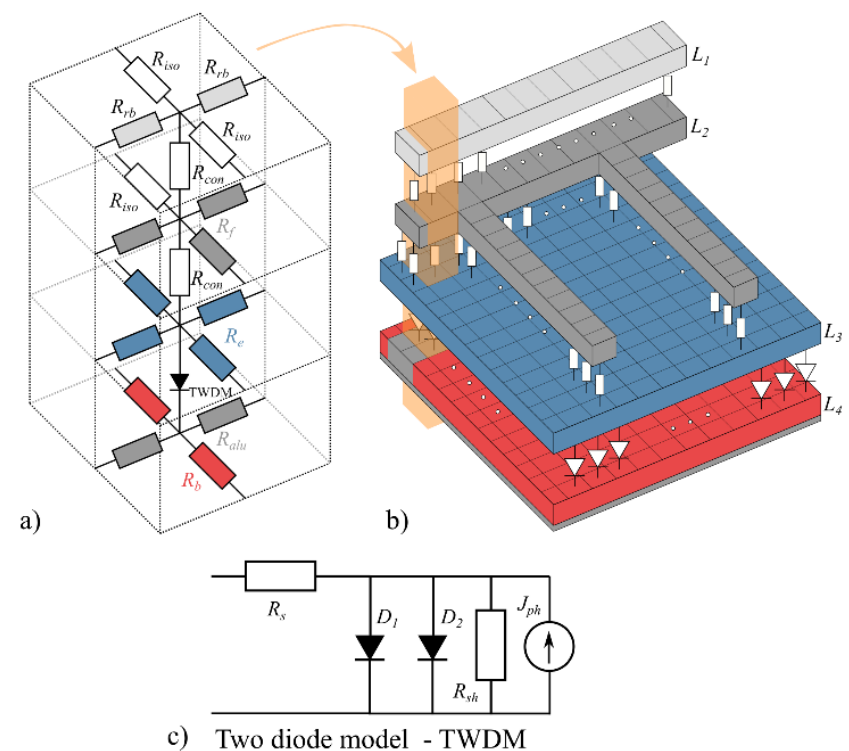

Figure 1. a) PVMOS model - element mesh, b) PVMOS model layer-by-layer (layer thicknesses are not shown to scale), and c) A two-diode model modelling a p-n junction.

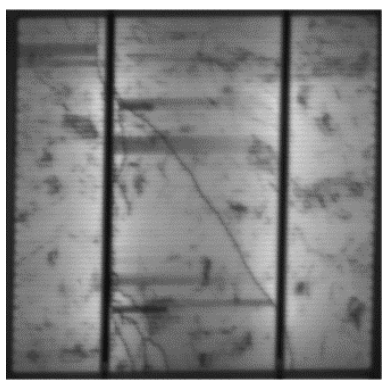

a)

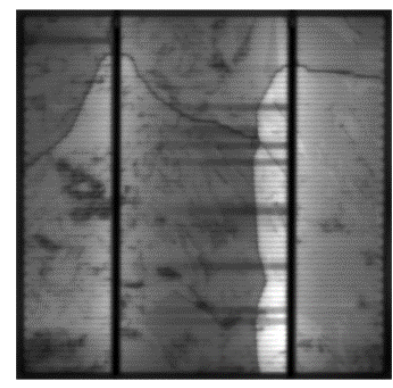

b)

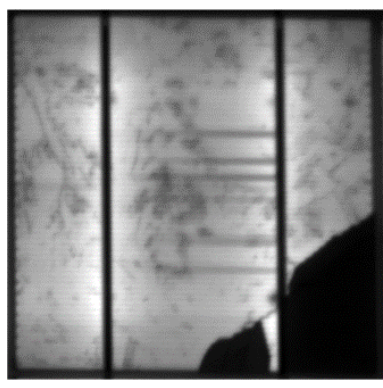

c)

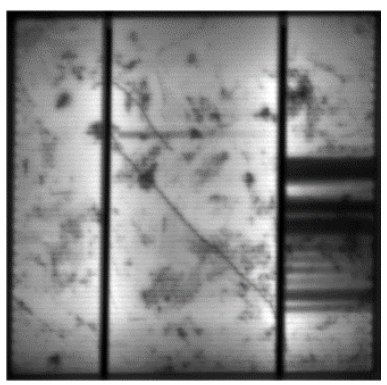

d)

Figure 2. Our classification of stress induced inhomogeneities and classification according to IEC 60904-13 [10]. a) Inactive crack (IEC crack mode A), b) Active crack (IEC crack mode B), c) Dead cell area (IEC crack mode C), and d) Contact finger interruption (IEC: Missing, broken or delaminated grid fingers). 

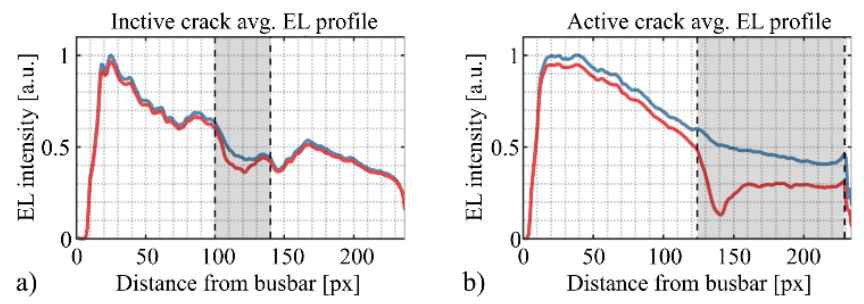

$$
\text { - Healthy _ Damaged }
$$

$$
\square \text { Affected area }
$$

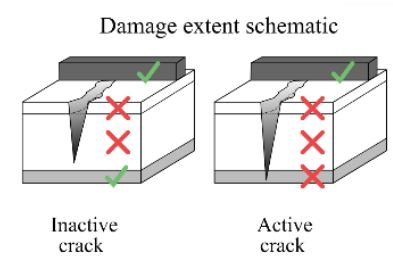

c)

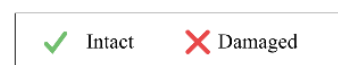

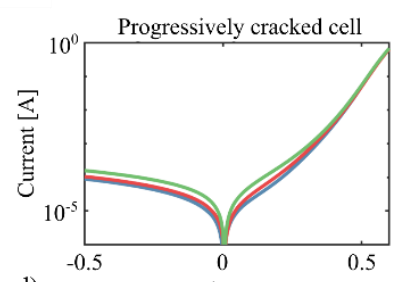

d)

Figure 3. Active and inactive crack manifestation. a) Inactive crack EL profile line scan, b) Active crack EL profile line scan, c) Inactive and active crack - damaged layers, and d) Effects of inactive cracks on the dark $I-V$ curve.

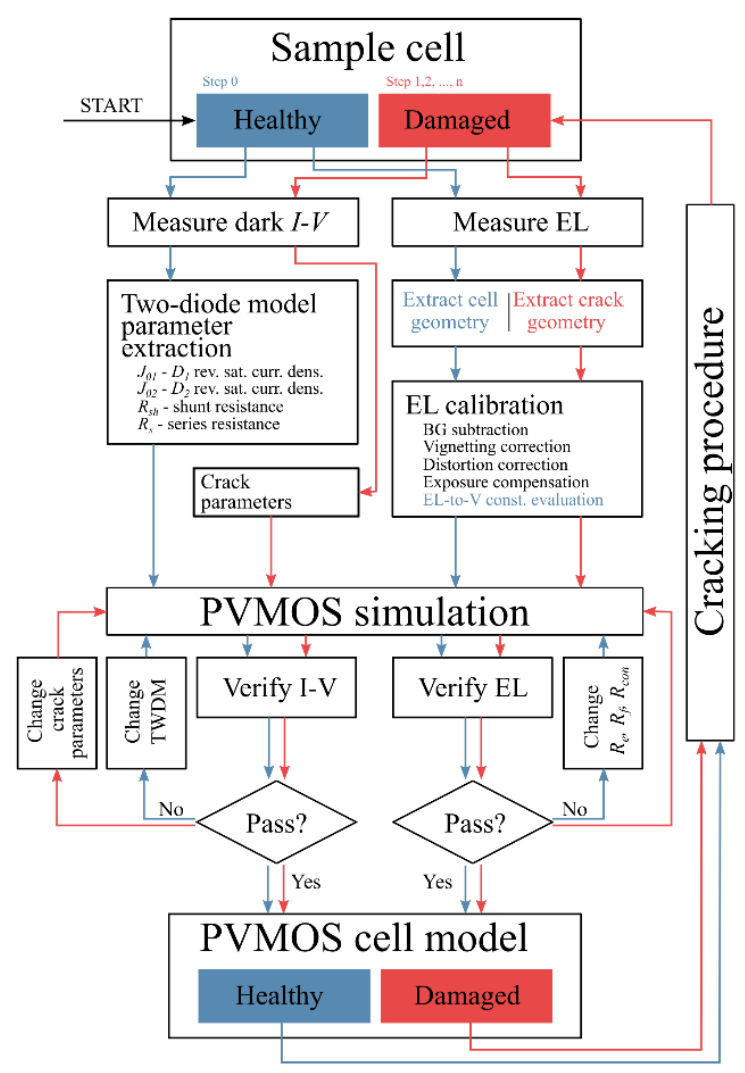

Figure 4. Experimental workflow - measurement, image correction, model calibration. 

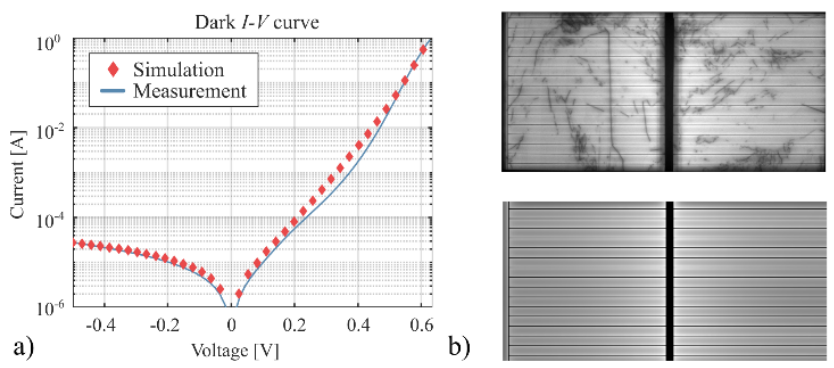

b)
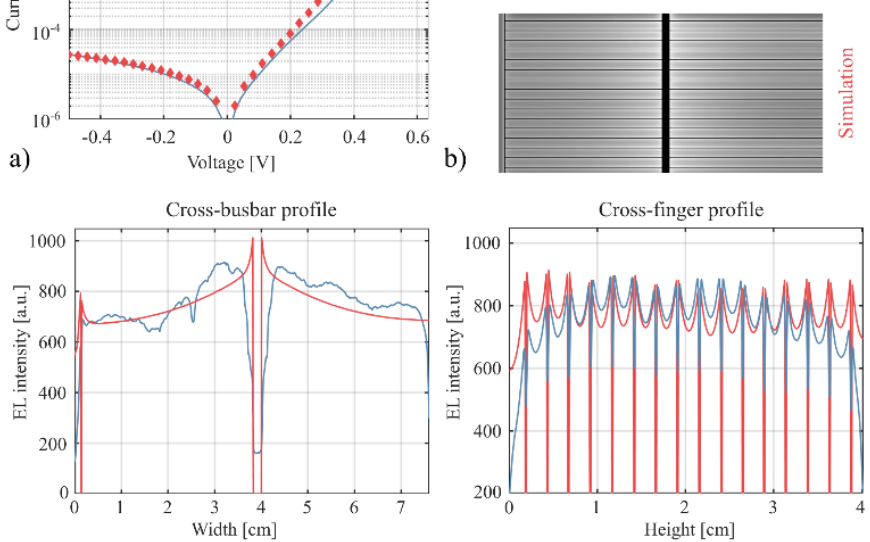

c) $\quad$ - Measurement - Simulation

d) $\quad$ - Measurement - Simulation

Figure 5. Results of healthy cell model simulation. a) Dark $I-V$ curve match, b) Comparison between the measured and the simulated EL image, c) Cross-busbar EL profile match, and d) Crossfinger EL profile match at Isc injection level.
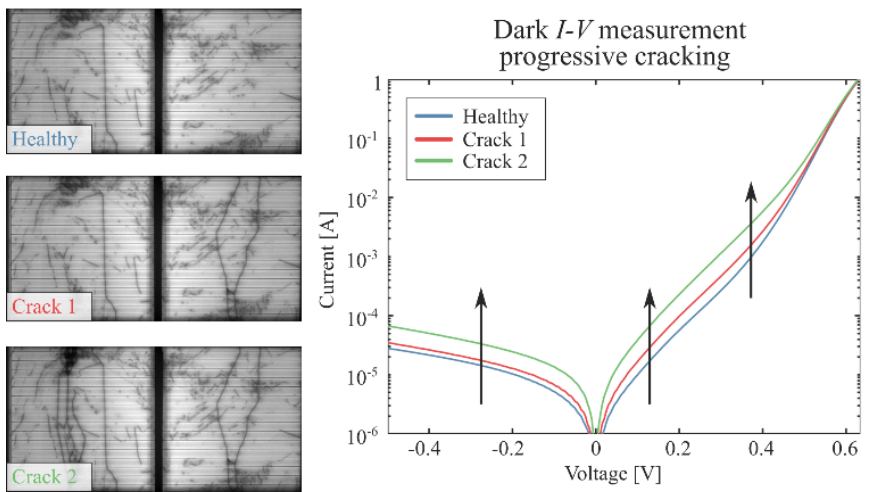

a)

b)

Figure 6. Measurement of a progressively damaged sample cell. a) Measured EL images, b) Measured dark $I-V$ curves. 


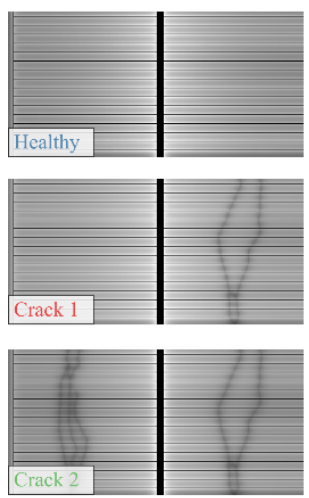

a)

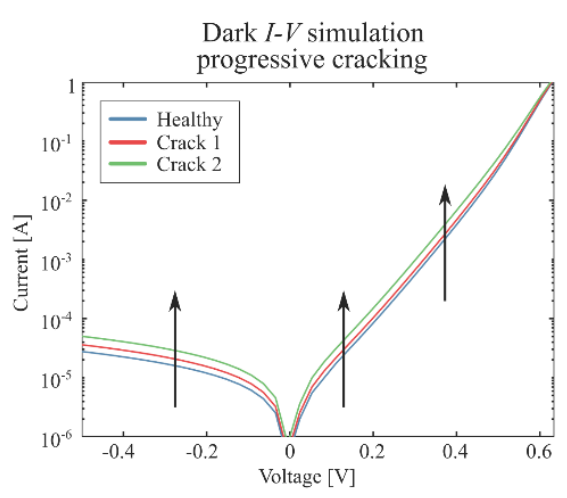

b)

Figure 7. Simulation of a progressively damaged sample cell. a) Simulated EL images, b) Simulated dark $I-V$ curves.

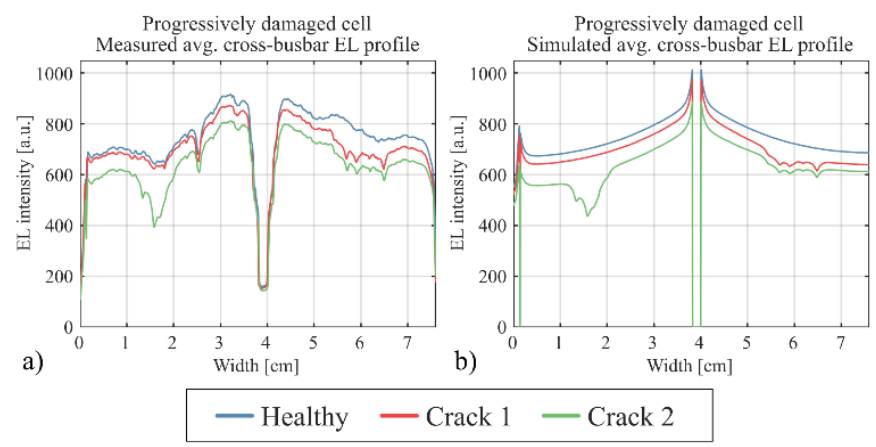

Figure 8. Comparison between measured and simulated cross-busbar EL profiles. a) Measured average cross-busbar EL profiles of a progressively cracked cell, b) Simulated average crossbusbar EL profiles of a progressively damaged cell. 
a)
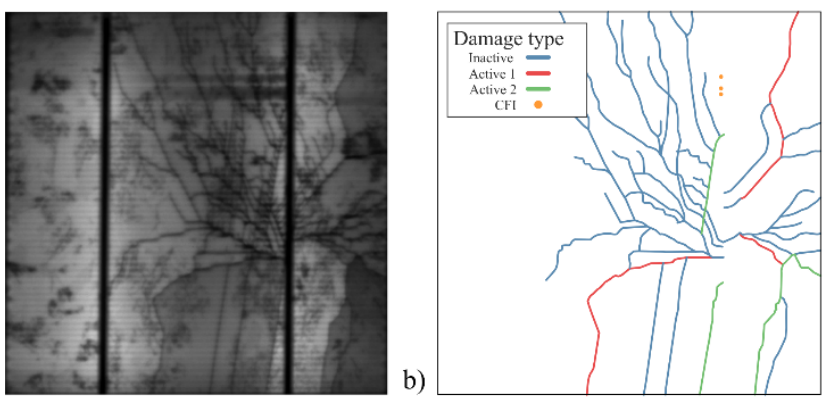

c)

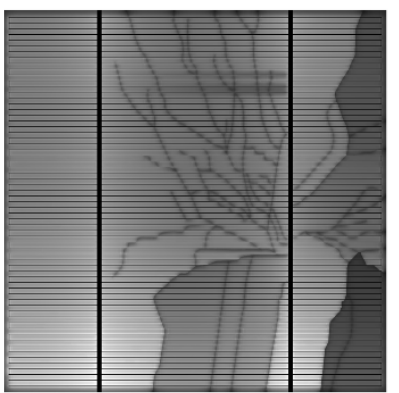

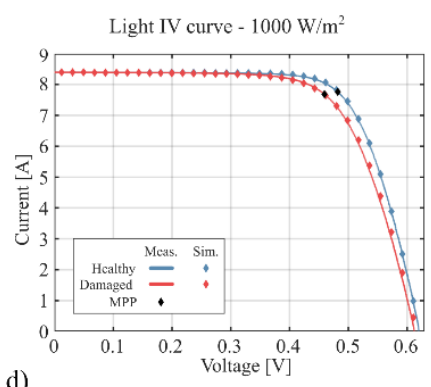

Figure 9. Simulation of a complexly damaged cell of a 2010 Bisol BMU 233 module. a) Measured EL image, b) Stress-induced inhomogeneties mask used in simulations with classification of inhomogeneities, c) Simulated EL image, and d) Healthy and damaged light $I-V$ curves comparison between the measurement and the simulation. 